\title{
The structural and accessory proteins $M$, ORF 4a, ORF 4b, and ORF 5 of Middle East respiratory syndrome coronavirus (MERS-CoV) are potent interferon antagonists
}

\author{
Yang Yang $^{1^{*}}$, Ling Zhang ${ }^{1^{*}}$, Heyuan Geng ${ }^{1^{*}}$, Yao Deng ${ }^{1}$, Baoying Huang ${ }^{1}$, Yin Guo ${ }^{2}$, Zhengdong Zhao ${ }^{3 \bowtie}$, \\ Wenjie $\operatorname{Tan}^{1}$ \\ ${ }^{1}$ Key Laboratory of Medical Virology, Ministry of Health, National Institute for Viral Disease Control and Prevention, Chinese \\ Center for Disease Control and Prevention, Beijing 102206, China \\ ${ }^{2}$ Institute of Materia Medica (IMM), Chinese Academy of Medical Sciences and Peking Union Medical College, Beijing 100050, \\ China \\ ${ }^{3}$ Key Laboratory of Pathogen System Biology, Ministry of Health, Institute of Pathogen Biology, Chinese Academy of Medical \\ Sciences, Beijing 100176, China \\ \ Correspondence: tanwj28@yahoo.cn (W. Tan), timjszzd@163.com (Z. Zhao)
}

Received September 29, 2013 Accepted November 1, 2013

\section{ABSTRACT}

The newly emerged Middle East respiratory syndrome coronavirus (MERS-CoV) is a highly pathogenic respiratory virus with pathogenic mechanisms that may be driven by innate immune pathways. The goal of this study is to characterize the expression of the structural (S, E, M, N) and accessory (ORF 3, ORF 4a, ORF 4b, ORF 5) proteins of MERS-CoV and to determine whether any of these proteins acts as an interferon antagonist. Individual structural and accessory protein-coding plasmids with an $\mathrm{N}$-terminal HA tag were constructed and transiently transfected into cells, and their native expression and subcellular localization were assessed using Western blotting and indirect immunofluorescence. While ORF $4 \mathrm{~b}$ demonstrated majorly nuclear localization, all of the other proteins demonstrated cytoplasmic localization. In addition, for the first time, our experiments revealed that the M, ORF 4a, ORF 4b, and ORF 5 proteins are potent interferon antagonists. Further examination revealed that the ORF 4a protein of MERS-CoV has the most potential to counteract the antiviral effects of IFN via the inhibition of both the interferon production (IFN- $\beta$ promoter activity, IRF-3/7 and NF-kB activation) and ISRE promoter element signaling pathways. Together, our results provide new insights into the function and pathogenic role of the structural and accessory proteins of MERS-CoV.

*These authors contributed equally to the work.

(C) Higher Education Press and Springer-Verlag Berlin Heidelberg 2013
KEYWORDS MERS-CoV, structural proteins, accessory proteins, interferon antagonists

\section{INTRODUCTION}

The 2002-2003 outbreak and spread of severe acute respiratory syndrome, which was caused by an emerging novel coronavirus (CoV) named severe acute respiratory syndromeassociated virus (SARS-CoV), eventually killed 774 of the $\sim 8000$ sickened individuals and caused world-wide economic damage in the range of 100 billon US dollars. A novel lineage $C$ beta-coronavirus was recently identified. The virus, renamed Middle East respiratory syndrome coronavirus (MERS-CoV), causes acute pneumonia with renal failure and was first identified in humans in the Middle East in 2012 (Zaki et al., 2012; de Groot et al., 2013). Through August 1, 2013, a total of 94 cases, including 46 fatalities, have been reported, and recent epidemiological investigations strongly suggest the possibility of human-to-human transmission (Breban et al., 2013; World Health Organization, 2013). These findings have reignited public concern about the potential for a MERS pandemic.

The MERS-CoV genome is approximately $30 \mathrm{~kb}$ in size and generally encodes three broad protein classes (van Boheemen et al., 2012) (Fig. 1A). Its virions are approximately 90 to $120 \mathrm{~nm}$ in diameter and contain a lipid bilayer surrounding a helical nucleocapsid structure that protects the genome. Several struc- 

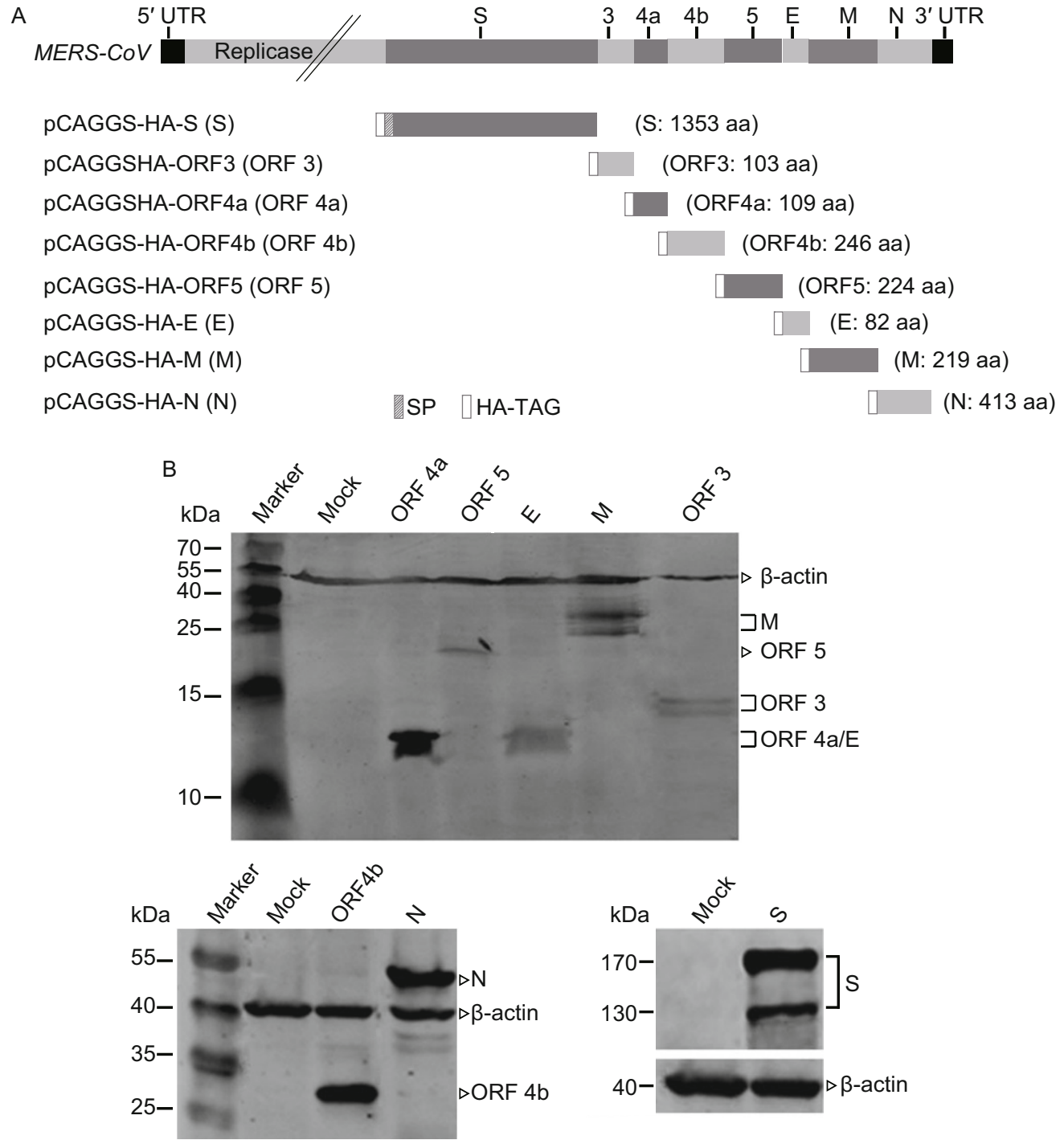

Figure 1. Construction and expression analysis of the individual structural and accessory proteins of MERS-CoV. (A) Schematic diagram of the genome organisation of SARS-CoV and MERS-CoV and expression constructs in this study. The genome structure of coronaviruses is highly conserved among all known coronaviruses. Each coronavirus has similar structural ORFs in their genomes. The accessory ORFs are unique to each coronavirus. There is no sequence or structural similarities between the MERS-CoV and SARS-CoV accessory proteins. The chemically synthesized coding genes of individual structural and accessory proteins were inserted into the expression vector pCAGGS with an HA-tag at the N-terminus of the protein. The SP (signal peptide) sequence of spike was also indicated. (B) Expression analysis of the individual structural and accessory proteins of MERS-CoV in 293T cells using Western blotting. Samples were collected from cells transiently transfected with individual ORF expression plasmids or pCAGGS (indicated as Mock) at 3 days posttransfection, and $100 \mathrm{ng}$ of protein was loaded into each lane for SDS-PAGE and Western blotting using mouse anti-HA and anti- $\beta$-actin mAbs as the primary antibodies at a 1:1000 dilution. Samples were normalized by measuring the relative amount of $\beta$-actin in the same sample.

tural proteins are encoded within the intact virion, including the spike $(\mathrm{S})$ protein, a nucleocapsid $(\mathrm{N})$ protein, an envelope $(\mathrm{E})$ protein, and the membrane $(\mathrm{M})$ protein. The $\mathrm{S}$ protein of MERS-CoV is a heavily glycosylated type I membrane protein that decorates the outside of the virion and is essential for attachment to the host receptor. For MERS-CoV, Dipeptidyl peptidase 4 (DPP4) is the receptor, while for SARS-CoV, the receptor is angiotensin-converting enzyme 2 (ACE2) (Raj et al., 2013). A second class of genes encodes the accessory or group-specific proteins. Each coronavirus genome encodes a distinct set of accessory proteins that are somehow important in the virus life cycle. SARS-CoV encodes ORFs 3a, 3b, 6, 7a, $7 \mathrm{~b}, 8$, and $9 \mathrm{~b}$ as well as an ORF protein internal to $\mathrm{N}$ (Marra et al., 2003). In the case of MERS-CoV, ORF 3, ORF 4a, ORF 
$4 \mathrm{~b}$, and ORF 5 are predicted to be accessory proteins (van Boheemen et al., 2012). These accessory proteins may influence viral pathogenesis and disease outcomes, regulate specific virus-host interactions and/or promote the development of an intracellular environment that is conducive for efficient virus growth, as reported for SARS-CoV and other coronaviruses (McBride et al., 2012). However, the precise location and function of these proteins remain unclear and are of particular interest for understanding the pathogenesis of MERS-CoV. The final broad category of coronavirus genes encodes the replicase proteins, also called nonstructural proteins. These viral proteins are encoded in the 5'-most two-thirds of the coronavirus genome, which is essential for polyprotein processing, replicase complex formation, and efficient virus replication.

Coronaviruses, similar to many other viruses, have evolved mechanisms to circumvent the innate immunity of their hosts at multiple levels (Totura et al., 2012). The principal response of mammalian cells to viral infection is the activation of the type I interferon (IFN)-mediated innate immune response through the production of type I IFNs (IFN- $\alpha$ and IFN- $\beta$ ). The subversion of the host IFN system is a key component of pathogenic viral infection and is mediated by virus-encoded IFN antagonist proteins. Each protein blocks one or more key signaling proteins in the IFN and NF-KB pathways to enhance viral replication and pathogenesis (García-Sastre et al., 2006; Randall et al., 2008; Taylor et al., 2013). A number of coronavirus gene products have been implicated in the disruption of signal transduction events required for the IFN response (Totura et al., 2012). Each protein antagonizes the innate immune response but uses different tools and targets to achieve these goals (GarcíaSastre et al., 2006).

The goal of this study is to characterize the expression and subcellular location of individual structural and accessory proteins of MERS-CoV and determine whether any of these proteins acts as an interferon antagonist. First, we analyzed the expression and subcellular localization of the individual proteins in cells that had been transiently transfected with structural and accessory protein-expressing plasmids. For the first time, our experiments revealed that the M, ORF 4a, ORF $4 \mathrm{~b}$, and ORF 5 proteins of MERS-CoV are potent interferon antagonists. Further examination revealed that the ORF $4 a$ protein of MERS-CoV has the most potential to act as an antagonist of the antiviral activity of IFN via the inhibition of both the interferon production (IFN- $\beta$ promoter activity, IRF-3/7 and NF-KB activation) and the ISRE promoter element signaling pathways.

\section{RESULTS}

Characterization of the expression and subcellular localization of MERS-CoV structural and accessory proteins

To characterize the expression and subcellular localization of the structural and accessory proteins of MERS-CoV, individual structural protein (S, E, M, and $\mathrm{N}$ ) and accessory protein (ORF 3 ,
ORF 4a, ORF 4b, and ORF 5) encoding plasmids were constructed with an $\mathrm{HA}$-tag at the $\mathrm{N}$-terminus of each protein (Fig. 1A). Individual expression plasmids were transiently transfected into 293T or BHK cells, and their native expression and subcellular localization were assessed using Western blotting and an indirect immunofluorescence assay (IFA) (Figs. 1B and 2).

As measured by Western blotting using a mAb against the $\mathrm{HA}$ tag and $\beta$-actin as the sample loading control, individual expression constructs encoding structural and accessory proteins of MERS-CoV were expressed at different levels at the appropriate size, as expected (Fig. 1B). The results also indicated that the expressions of S, E, M, ORF 3, and ORF 4a experience some post-translational modifications that need further confirmation, as S, M, ORF 3, and ORF 4a were predicted to have N-glycosylation sites.

Post-translational modifications and the correct subcellular localization of viral structural proteins are prerequisites for the assembly and budding of enveloped viruses. Coronaviruses, such as the SARS-CoV bud from the endoplasmic reticulumGolgi intermediate compartment (Lai et al., 2006). In this study, the subcellular distribution of MERS-CoV surface proteins (S, $\mathrm{E}$, and $\mathrm{M}$ ) and $\mathrm{N}$ protein were analyzed by IFA and confocal microscopy using N-terminally tagged proteins (Fig. 2A-D).

We next analyzed the expression of MERS-CoV surface proteins. S protein expression was detected throughout the cytoplasm and at the plasma membrane; further analysis showed that it co-localized with ER and Golgi markers and partially colocalized with ERGIC in compartments along the secretory pathway (Fig. 2A). Previous studies showed that coronavirus $\mathrm{E}$ proteins may have different subcellular localizations. For example, the SARS-E protein forms large membrane clusters codistributing with ER markers, while the IBV-E protein is localized to the Golgi complex (Corse et al., 2000; Nal et al., 2005; Nieto-Torres et al., 2011). Our results demonstrated that the MERS-E protein mainly co-localizes with the Golgi apparatus, similar to IBV-E (Fig. 2B). The M proteins of several coronaviruses have been reported to accumulate in the Golgi complex of mammalian host cells (Nal et al., 2005). Likewise, the MERS-CoV M protein clearly co-localized with the Golgi apparatus and ERGIC in the perinuclear area, and there were also distinct punctate within the cytoplasm (Fig. 2C). In addition, we observed that the MERS-CoV N protein remained exclusively localized to the cytoplasm, with no significant co-localization with the ER, ERGIC, or Golgi apparatus (Fig. 2D).

Previous studies have showed SARS-CoV accessory proteins have different subcellular localizations and functions (McBride et al., 2012). Similarly, MERS-CoV accessory proteins also have different subcellular localizations (Fig. 2E-H). ORF 3 demonstrated punctate cytoplasmic distribution and co-localization with the ER and ERGIC (Fig. 2E), while ORF 4a was detected throughout the cytoplasm without any significant colocalization with the ER, ERGIC, or Golgi (Fig. 2F). Similar to SARS-CoV ORF 3b, MERS-CoV ORF $4 b$ localized majorly to the nucleus (Fig. $2 \mathrm{G}$ ); its molecular determinants need further investigation (Yuan et al., 2005); finally, ORF 5 was found to be 


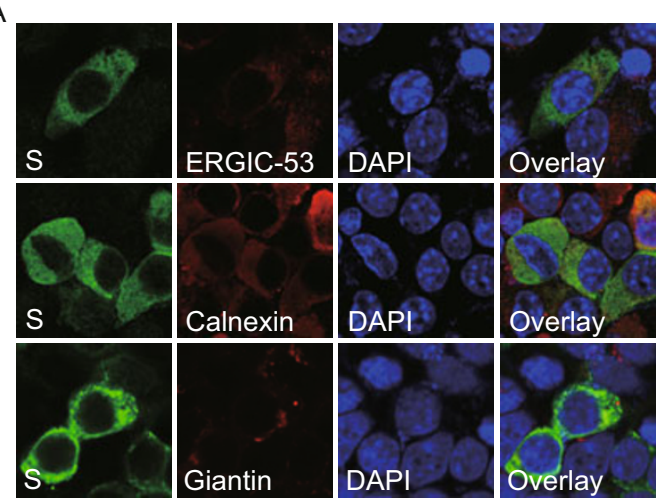

c

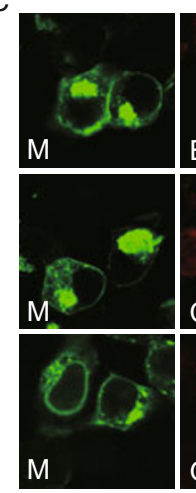

E
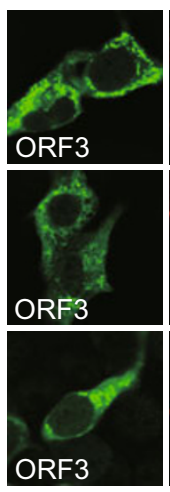

G
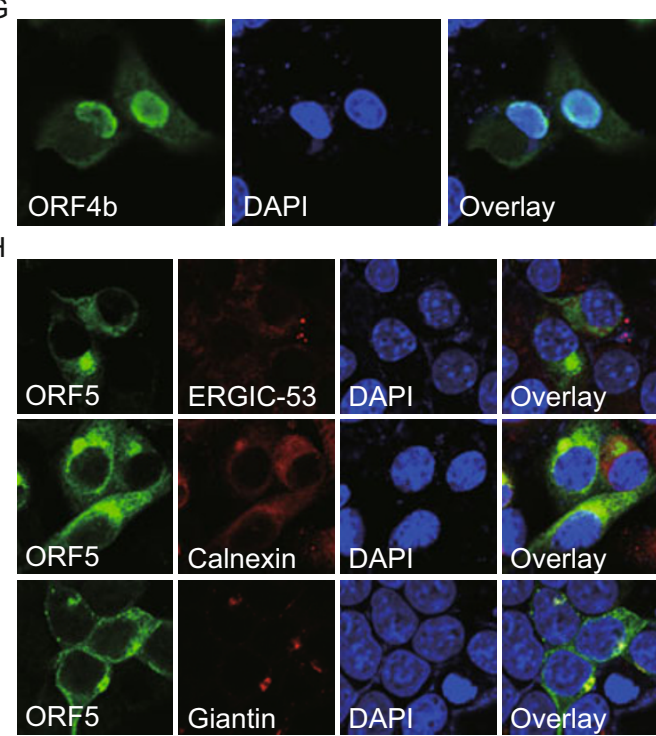

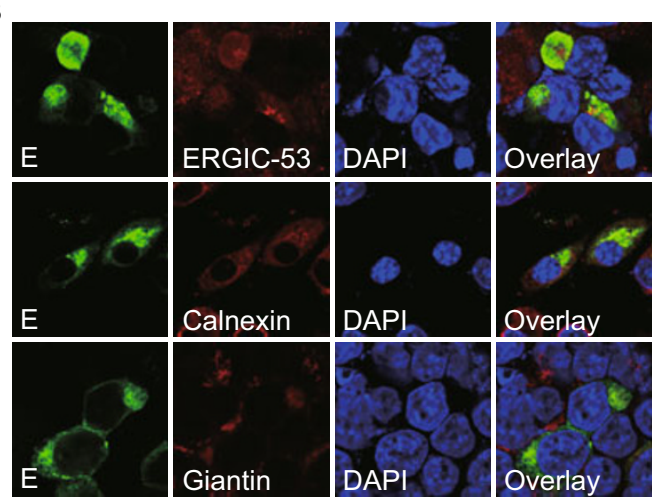

D
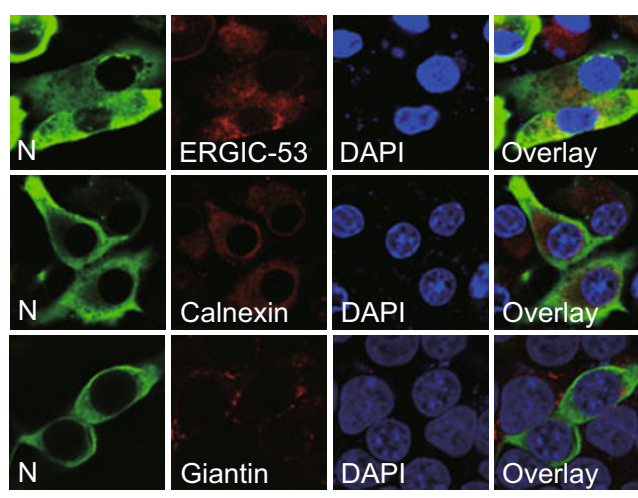

Overlay
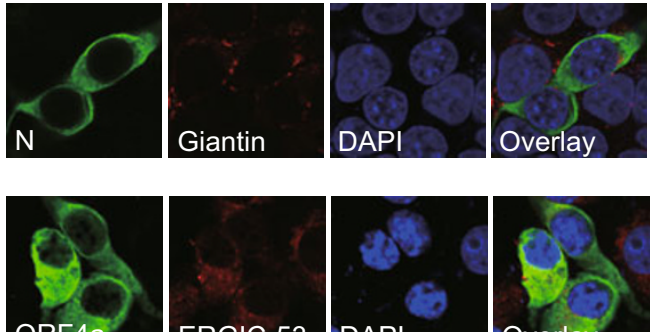

ORF4a
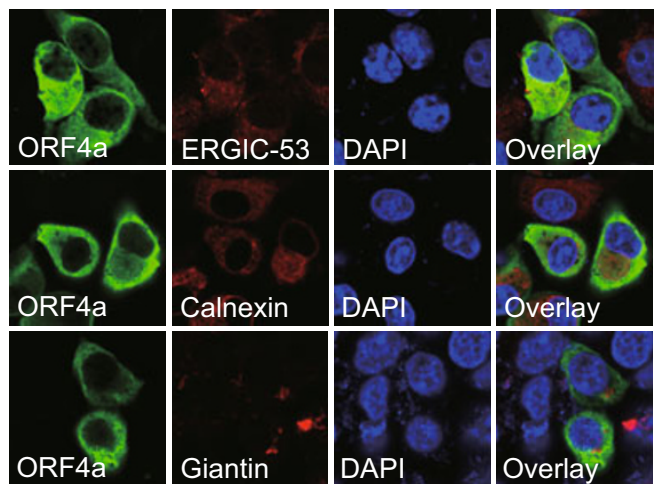

Figure 2. Confocal laser scanning microscopy of cells expressing individual recombinant MERS-CoV structural and accessory proteins costained with different antibodies for cellular organelles. Cells were fixed and dually labeled with antibodies against the MERS-CoV proteins and the corresponding cellular organelles at $24 \mathrm{~h}$ p.t. Cells were analyzed by confocal microscopy using a 100× objective, and representative images are shown. Left panels: staining with mouse anti-HA (MERS-CoV protein marker) and donkey anti-mouse-Alexa Fluor 488 (Molecular Probes). The second panels from top to bottom: co-staining of cellular organelles with goat anti-ERGIC53 (marker for the ER-Golgi intermediate compartment), goat anti-Calnexin (ER marker) or goat anti-Giantin (Golgi marker) together with donkey anti-goat Alexa Fluor 594 antibody. The third panels: staining with DAPI (Molecular Probes) to detect nuclei. The right panels show merged pictures, where yellow areas represent co-localization. Green represents MERS-CoV proteins, red represents the organelle marker protein, and blue represents chromatin. $S(A), E(B), M(C)$, and N (D); ORF $3(E)$, ORF 4a (F), ORF 4b (G), and ORF $5(\mathrm{H})$ are shown. 

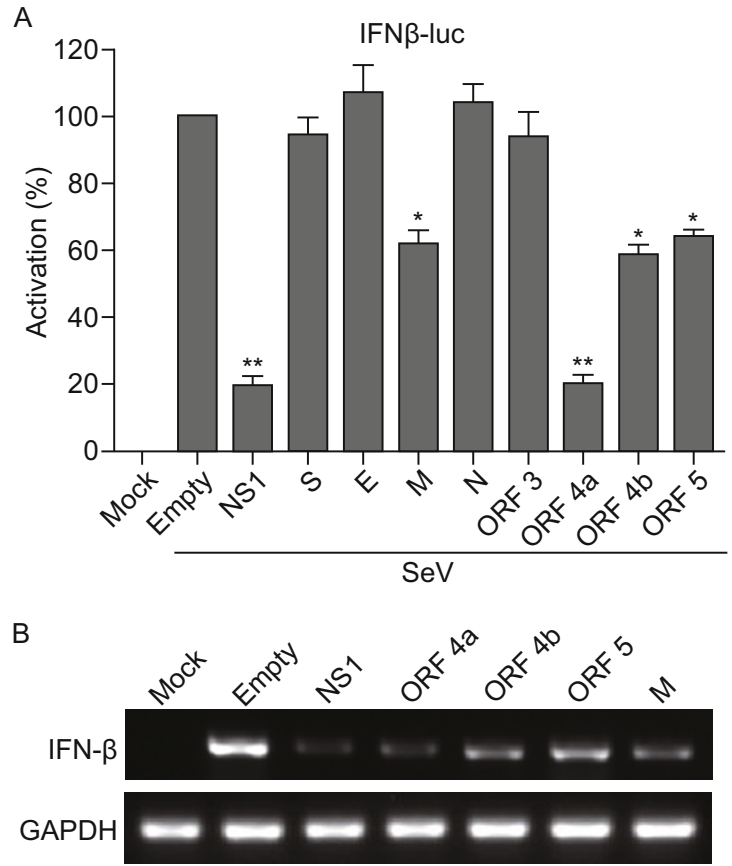

Figure 3. MERS-CoV ORF 4a, ORF 4b, ORF 5 , and M proteins inhibit the induction of the IFN- $\beta$. (A) 293T cells were co-transfected with $p G$ L3-IFN $\beta$-luc (a plasmid expressing Firefly luciferase under the control of the IFN- $\beta$ promoter), an internal control plasmid pRL-SV40 (a plasmid constitutively expressing Renilla luciferase) and the indicated plasmids. At $24 \mathrm{~h}$ post-transfection, cells were infected with Sendai virus. Cells were harvested at $24 \mathrm{~h}$ postinfection and analyzed for Firefly and Renilla luciferase. Data are representative of three independent experiments with triplicate samples. ${ }^{*}, P<0.05 ;{ }^{* *}, P<0.01$ versus empty (Student's $t$-test). (B) 293T cells were transfected with the indicated plasmids for $24 \mathrm{~h}$ and then infected with Sendai virus for $8 \mathrm{~h}$. Total cellular RNA was extracted and the expression of IFN- $\beta$ was measured by RTPCR. Glyceraldehyde 3-phosphate dehydrogenase (GAPDH) was used as a control to ensure that RNA of comparable quality was analyzed.

clearly co-localized with the Golgi apparatus and ERGIC in the perinuclear area (Fig. 2H).

ORF $4 a$, ORF $4 b$, ORF 5 , and $M$ proteins inhibit the induction of IFN- $\beta$

It has been previously reported that some of the SARS-CoV structural and accessory proteins display different interferon antagonist activities, so we sought to determine whether the MERS-CoV structural and accessory proteins have the same function (Kopecky-Bromberg et al., 2007; Freundt et al., 2009; Siu et al., 2009). Preventing expression from the interferon promoter is one of the mechanisms by which viral proteins inhibit the interferon response, so IFN- $\beta$ promoter activation in response to Sendai virus (SeV) infection in the presence or absence of MERS proteins was measured. As expected, the positive control (NS1) demonstrated the strongest inhibition of IFN- $\beta$-luciferase, up to approximately $80 \%$ (Fig. 3A). The MERS-CoV ORF 4a, ORF 4b, ORF 5, and M proteins all showed different levels of inhibition of IFN- $\beta$ promoter, of which ORF 4a was the strongest, as it exhibited a similar inhibition as the positive control (NS1). This observation was further confirmed at the mRNA level by RT-PCR analysis (Fig. 3B). These results indicate that the four proteins function as antagonists by inhibiting the induction of interferon.

ORF 4a, ORF 4b, ORF 5, and $M$ inhibit the function of IRF-3 and ORF $4 a$ inhibits the function of NF-KB

The expression of type I IFN is controlled by latent transcription factors, including the IFN regulatory factor 3 (IRF-3). IRF-3 is constitutively expressed in the cytoplasm in latent form and is regulated by posttranslational modification. Phosphorylated IRF-3 undergoes dimerization, nuclear translocation, and association with the co-activator $\mathrm{CBP} / \mathrm{p} 300$ and then primarily activates the IFN- $\beta$ promoter. Previous studies have shown that the binding of activated IRF-3 to the interferon promoter is necessary for interferon induction (García-Sastre et al., 2006). The ability of the four MERS-CoV proteins to block the IRF3-mediated activation of its binding sites was evaluated using a plasmid containing a firefly luciferase gene under the control of a promoter with three IRF-3 binding sites (55-CIB). All four MERS-CoV proteins, i.e., ORF $4 a$, ORF $4 b$, ORF 5, and M, inhibited the activation of the 55-CIB promoter similarly to the positive control, NS1 (Fig. 4A). These results indicate that ORF $4 a$, ORF $4 b$, ORF 5, and M prevent the activation of promoters requiring IRF-3 binding, such as the interferon promoter.

Given the ability of the four proteins to inhibit the IRF-3-mediated activation of its binding sites, it was important to determine how the function of IRF-3 was blocked. As expected, IRF-3 remained in the cytoplasm of mock cells, while it translocated to the nuclei of SeV-infected cells (Fig. 4B). Cells expressing MERS-CoV proteins demonstrated exclusively cytoplasmic GFP-IRF-3 localization following SeV infection, providing evidence that the expression of the four proteins blocks the virusinduced nuclear accumulation of IRF-3.

$\mathrm{NF}-\mathrm{KB}$ is another cellular component necessary for the production of interferon. Similar to IRF-3, NF-kB is a transcription factor that is activated after viral infection and binds to the interferon promoter to activate transcription (García-Sastre et al., 2006). The ability of the four proteins to block the NF-KBmediated activation of its binding sites was evaluated using a plasmid containing a firefly luciferase gene under the control of a promoter with two NF-kB binding sites. As expected, the positive control, NS1, inhibited expression from this promoter. Only the ORF 4a protein inhibited the activation of the NF-KB promoter (Fig. 4C), with an inhibition rate of approximate $50 \%$ $(P<0.05)$. This result may explain why ORF 4 a could inhibit the production of IFN- $\beta$ to a greater extent than the other three proteins. 


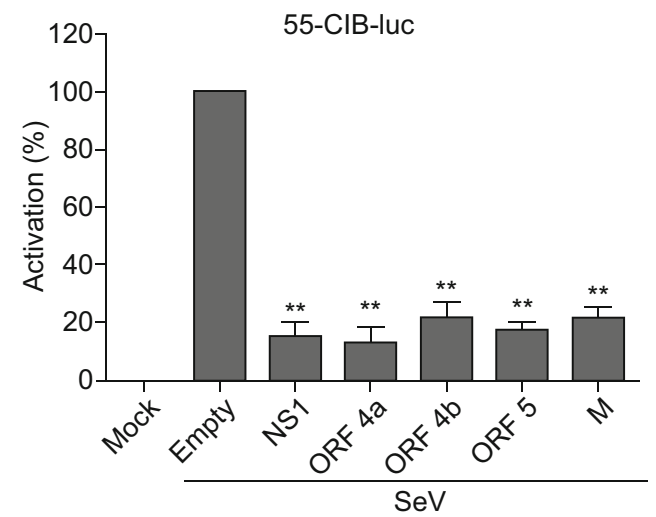

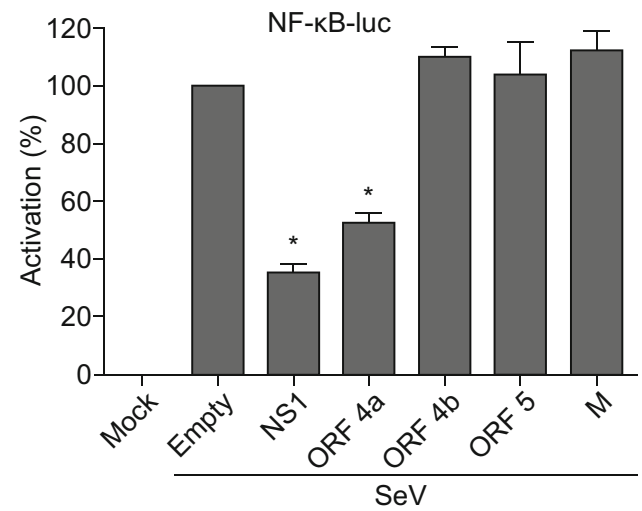

B

$$
\begin{gathered}
\text { MERS-CoV } \\
\text { proteins }
\end{gathered}
$$

\section{Empty \\ infected}

ORF $4 a$
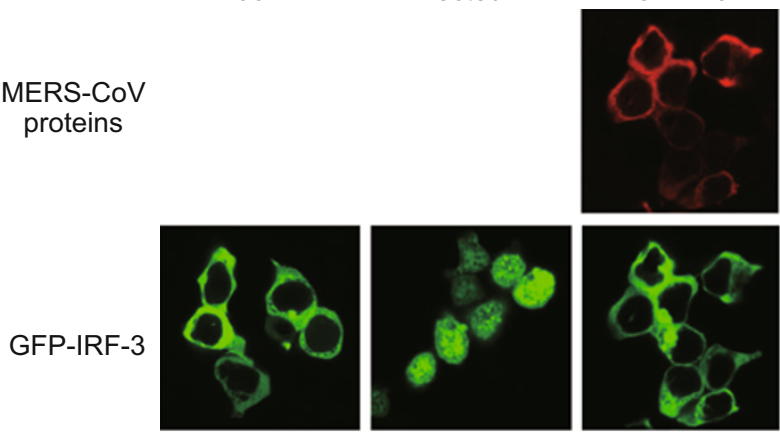

DAPI

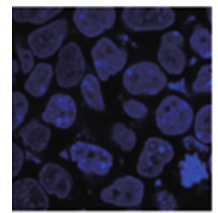

Overlay

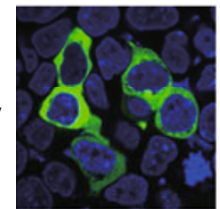

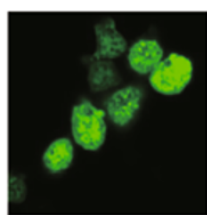
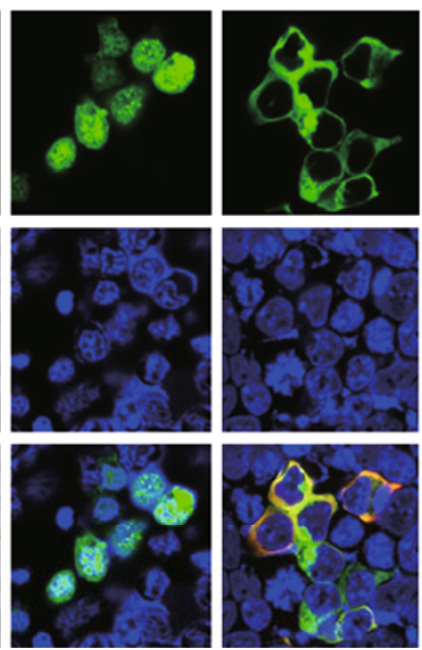

ORF 4b
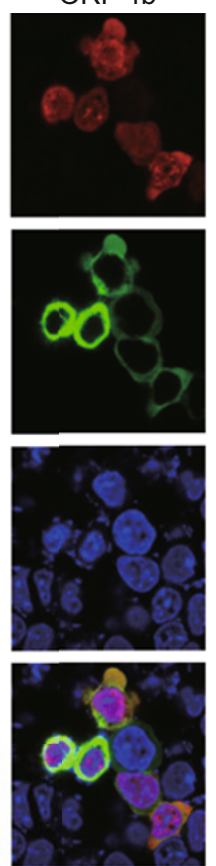

ORF 5
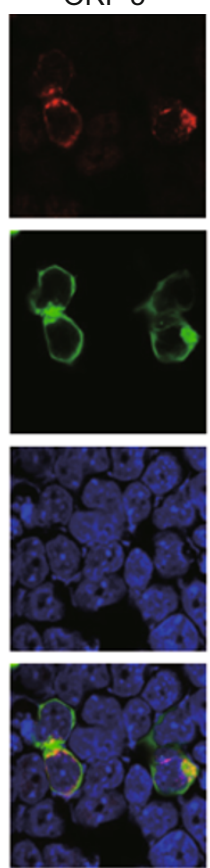

M
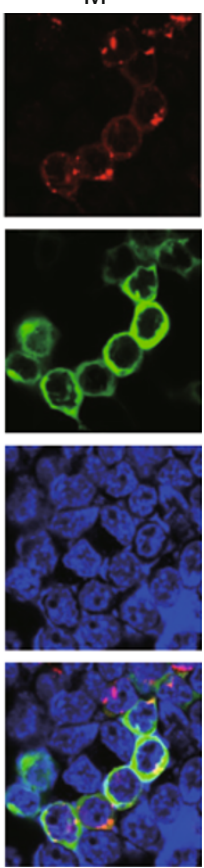

Figure 4. ORF 4a, ORF 4b, ORF 5, and M proteins inhibit the function of IRF-3, and ORF 4a protein inhibits NF-kB activation. (A) 293T cells were co-transfected with the 55-CIB promoter, which contains three IRF-3 binding sites, a plasmid that constitutively expresses Renilla luciferase, and plasmids expressing SARS-CoV proteins or the indicated control plasmids. Cells were infected with Sendai virus $24 \mathrm{~h}$ post-transfection. Cells were harvested at $24 \mathrm{~h}$ post-infection and analyzed for Firefly and Renilla luciferase. Data were normalized using the Renilla luciferase values. Data are representative of three independent experiments with triplicate samples. ${ }^{* *}, P<0.01$ versus empty vector (Student's $t$-test). (B) 293T cells were transfected with the indicated plasmids for $24 \mathrm{~h}$ and then infected with Sendai virus. Cells were fixed $16 \mathrm{~h}$ post-infection and analyzed by microscopy using an antibody that recognizes the HA tag and a TRITC-conjugated goat anti-mouse secondary antibody. (C) ORF 4a protein inhibits NF-kB activation. 293T cells were co-transfected with a plasmid expressing firefly luciferase under the control of an NF-kB-responsive promoter containing two NF-kB binding sites, the Renilla luciferase plasmid, and the indicated plasmids. At $24 \mathrm{~h}$ post-transfection, cells were infected with Sendai virus. Cells were harvested and analyzed for Firefly and Renilla luciferase activity at $24 \mathrm{~h}$ post-infection. Data were normalized using the Renilla luciferase values. Data are representative of three independent experiments with triplicate samples. ${ }^{*}, P<0.05$ versus empty vector (Student's $t$-test).

\section{ORF 4a, ORF 4b, and M proteins inhibit expression from the ISRE promoter}

The binding of secreted IFN to its receptor (IFNAR) and the subsequent activation of IFN-stimulated gene (ISG) induction is the second step of the biphasic IFN response. The secreted IFN from the infected cells binds to an interferon receptor and signals through a JAK/STAT pathway to activate ISGs containing an ISRE in the promoter regions, resulting in the induction of their transcription (García-Sastre et al., 2006). The ability of 
the four proteins to inhibit interferon signaling was evaluated using a plasmid containing a firefly luciferase gene under the control of an ISRE promoter. After treatment with IFN- $\beta$, the ORF $4 \mathrm{a}$, ORF $4 \mathrm{~b}$, and M proteins efficiently inhibited expression from the ISRE promoter to different extents $(P<0.05)$, up to $70 \%$ for ORF $4 a$. However, the ORF 5 protein had little effect on expression from the ISRE promoter (Fig. 5A). This observation was also confirmed at the mRNA level by detecting the expression of ISG54 and ISG56 using RT-PCR analysis (Fig. 5B). These data indicate that the ORF 5 protein inhibits only interferon synthesis, while the ORF $4 a$, ORF $4 b$, and M proteins inhibit not only interferon synthesis but also interferon signaling.

\section{DISCUSSION}

An understanding of how each antagonist affects the innate immune response illuminates key interactions between the host signaling pathway components and the virus. In addition, these studies pinpoint key host cell components that function to regulate viral replication and pathogenesis, providing novel targets for the development of antiviral compounds (García-Sastre et al., 2006; Randall et al., 2008; Taylor et al., 2013). In this study, we have used several IFN antagonist assays to identify the $M$, ORF $4 a$, ORF $4 b$, and ORF 5 proteins of MERS-CoV as potential IFN antagonists. These novel findings, coupled with the previous identification of differential IFN antagonist activities encoded within different regions of coronaviruses, suggest that Coronaviridae genomes likely encode multiple and different antagonists of the innate immune system. Understanding the mechanism of action of each antagonist may also direct us to novel therapeutic targets on both the virus and the host.

Our results showed that, similar to other coronaviruses, individually expressed recombinant MERS-CoV S protein was detected throughout the typical secretory pathway of mammalian cells, from the ER to the plasma membrane. Consistent with SARS-S, MERS-S may also contain a dibasic motif that retains the protein in the ERGIC (Lu et al., 2011). However, unlike the SARS-CoV S protein, the MERS-CoV S protein did not primarily concentrate in the Golgi region (Nal et al., 2005). Previous studies on SARS-CoV showed that the M protein localizes predominantly to the Golgi apparatus and overlaps with the ERGIC (Nal et al., 2005); our results showed the same localization for the MERS-CoV M protein. Whereas SARS-CoV $E$ is generally detected in the perinuclear regions and the $E R$ (Nieto-Torres et al., 2011), MERS-CoV E was predominantly detected in the Golgi apparatus in this study, similarly to the IBV-E protein (Corse et al., 2000). While the N protein of several CoVs localizes to the nucleolus during infection and after the transfection of cells with $\mathrm{N}$ genes, such as IBV, TGEV, and MHV (Hiscox et al., 2001; Lontok et al., 2004), the $\mathrm{N}$ protein of MERS-CoV was observed to exclusively localize to the cytoplasm, similar to the $\mathrm{N}$ protein of SARS-CoV (Wurm et al., 2001; Surjit et al., 2005; You et al., 2005). MERS-N is predicted to contain two nuclear localization signals (pat 4: RHKR at 251 aa; pat7: PKKEKKQ at 362 aa) using PSORTII (http://psort.nibb.

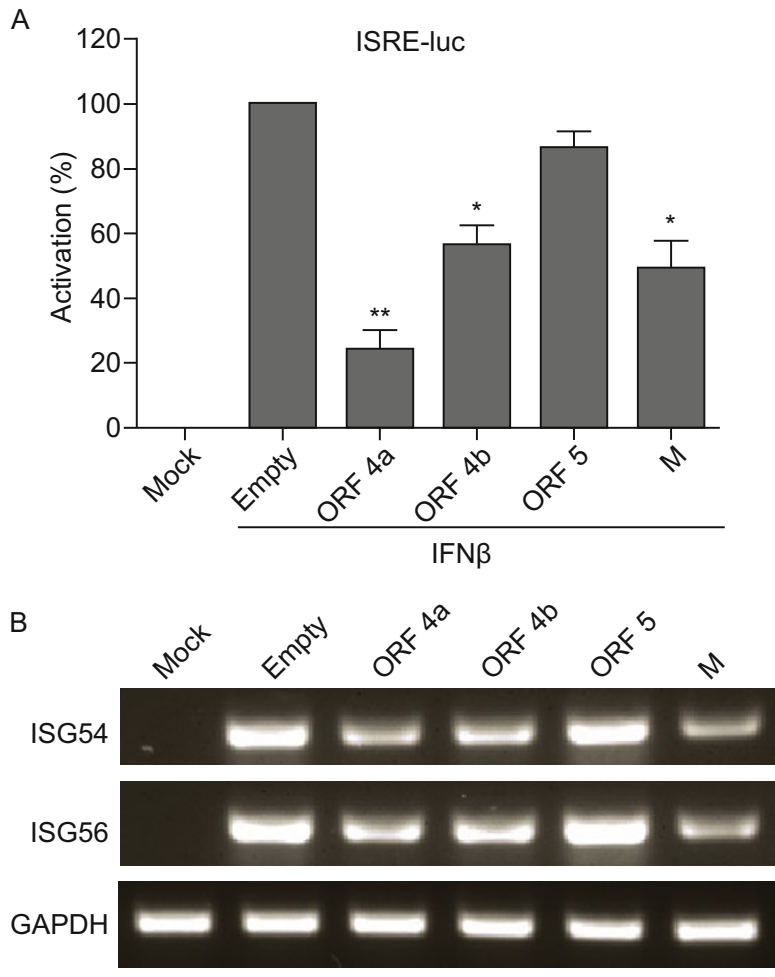

Figure 5. ORF 4a, ORF 4b, and M proteins inhibit ISRE activation. (A) 293T cells were co-transfected with a plasmid expressing firefly luciferase under the control of an ISRE promoter, the Renilla luciferase plasmid and the indicated plasmids. At $24 \mathrm{~h}$ post-transfection, cells were treated with $3000 \mathrm{U} / \mathrm{mL}$ exogenous IFN- $\beta$. Cells were harvested and analyzed for Firefly and Renilla luciferase activity at $24 \mathrm{~h}$ post-infection. Data were normalized using the Renilla luciferase values. Data are representative of three independent experiments with triplicate samples. *, $P<0.05$; ${ }^{* *}$, $P<0.01$ versus empty vector (Student's $t$-test). (B) 293 T cells were transfected with the indicated plasmids for $24 \mathrm{~h}$ and then infected with Sendai virus for $8 \mathrm{~h}$. Total cellular RNA was extracted and the expression of ISG54 and ISG56 were measured by RTPCR. Glyceraldehyde 3-phosphate dehydrogenase (GAPDH) was used as a control to ensure that RNA of comparable quality was analyzed.

ac.jp). However, the protein does not seem to localize to the nucleus, so we may presume that the two nuclear localization signal (NLS) motifs are inactive or inaccessible or that cytoplasmic localization was the dominant localization signal in the protein. These data suggest that the nuclear localization of the $\mathrm{N}$ protein is not a common property among all coronaviruses.

Although accessory proteins from coronaviruses are often dispensable for viral replication, they may play vital roles in virulence and pathogenesis by affecting host innate immune responses, encoding pro- or anti-apoptotic activities or impacting other signaling pathways that might influence disease outcomes (Susan et al., 2011). To date, little is known about the subcellular localization and function of MERS-CoV accessory 
proteins. Among the four accessory proteins of MERS-CoV, the localization of ORF $4 \mathrm{~b}$ is of particular interest, as it majorly localizes to the nucleus, similar to the SARS-CoV ORF $3 b$ (Yuan et al., 2005). Previous studies have shown the molecular determinants for nuclear localization and the possible functions of ORF 3b, such as the induction of apoptosis, cell $\mathrm{G}_{0} / \mathrm{G}_{1}$ arrest and IFN antagonism (Yuan et al., 2005; Khan et al., 2006; Kopecky-Bromberg et al., 2007; Freundt et al., 2009). In our study, we showed that ORF $4 \mathrm{~b}$ could act as an IFN antagonist, though the molecular determinants for its nuclear localization and other possible functions need further investigation.

The initial host response to infection is controlled by the innate immune system, which serves as a robust barrier to the establishment and maintenance of a productive viral infection. However, viruses have coevolved an armament of components that evade and/or block host innate immune responses, or they express components that shield infection from detection (García-Sastre et al., 2006; Randall et al., 2008; Taylor et al., 2013). Type I IFNs are essential for host protection against coronaviruses. The viruses may encode proteins (IFN antagonists) that directly inhibit the signaling pathways that are responsible for IFN induction. Significant amounts of data support the hypothesis that coronaviruses encode one or more IFN antagonist genes. Among the human coronaviruses, SARS-CoV is the best characterized both biochemically and molecularly. Notably, the first coronavirus antagonists of innate immunity were documented in the SARS-CoV genome, pioneering similar studies in other important human and animal CoV genomes. Until recently, SARS-CoV has been shown to have at least 8 proteins that inhibit IFN induction/signaling, including nsp1 (Wathelet et al., 2007; Kamitani et al., 2009; Huang et al., 2011), PLP (Devaraj et al., 2007; Frieman et al., 2009; Sun et al., 2012), nsp7, nsp15 (Frieman et al., 2009), ORF 3b, ORF 6, N, and M (Kopecky-Bromberg et al., 2007; Siu et al., 2009; Lu et al., 2011). Moreover, the SARS-CoV ORF 6 protein is known to inhibit IFN-induced JAK-STAT signaling by blocking the nuclear translocation of phosphorylated STAT1 (p-STAT1) (Frieman et al., 2007; Kopecky-Bromberg et al., 2007). Recent studies have found that the MERS-CoV failed to elicit strong type I IFN or proinflammatory innate immune responses in ex vivo respiratory tissue cultures. The replication of MERS-CoV was strongly inhibited by treatment with cyclosporin A or IFN- $\alpha$, and MERS-CoV infection could not inhibit the nuclear translocation of p-STAT1 (Chan et al., 2013; de Wilde et al., 2013), indicating that MERS-CoV may have evolved some alternative mechanisms to combat IFNs.

Similar to SARS-CoV (Totura et al., 2012), several MERSCoV structural and accessory proteins act as IFN antagonists when transiently expressed in this study. The $M$ protein and the products of the accessory genes ORF $4 a$, ORF $4 b$, and ORF 5 were all found to prevent the synthesis of IFN- $\beta$ through the inhibition of interferon promoter activation and IRF-3 function. The possible mechanism for the inhibition of IRF-3 nuclear translocation is the inhibition of the phosphorylation of IRF-3 (García-Sastre et al., 2006). In addition, the ORF 4a, ORF 4b, and $\mathrm{M}$ proteins inhibited ISRE activation. Furthermore, ORF 4a protein inhibited NF-KB and was the most potent interferon antagonist among all above proteins tested. Because MERSCoV infection could not inhibit the nuclear translocation of $p$ STAT1 (de Wilde et al., 2013), the mechanism of the inhibition of interferon signaling for ORF $4 a$, ORF $4 b$, and M needs further elucidation. Similar to other highly pathogenic viruses, such as the Nipah virus (Shaw et al., 2009), MHV (Ye et al., 2007; Koetzner et al., 2010; Zhao et al., 2011), SARS-CoV (Devaraj et al., 2007; Frieman et al., 2007; Kopecky-Bromberg et al., 2007; Frieman et al., 2009; Siu et al., 2009; Lu et al., 2011; Sun et al., 2012), and Ebola viruses (Reid et al., 2006; Basler et al., 2007), MERS-CoV encodes more than one protein that is able to inhibit interferon, which may contribute to the severe pathogenicity of the virus. Given the diverse array of novel genetic functions, it is not surprising that MERS-CoV also encodes several proteins that modulate the host innate immune response during infection via different pathways and acts on multiple targets within the innate immune response (Frieman et al., 2008; Totura et al., 2012).

As reported for other viruses, the MERS-CoV/host interaction and the modulation of the innate immune response are highly dependent on cell type, virus concentration, and whether the results are obtained from in vitro or in vivo experimentation (García-Sastre et al., 2006; Randall et al., 2008; Totura et al., 2012; Taylor et al., 2013). Our primary studies on MERS$\mathrm{CoV}$ were performed using over-expression constructs in cell culture using individual viral components, a system that may not accurately reflect the innate immune signaling that occurs during virus infection in vivo. Additional studies are needed to elucidate the potential for IFN antagonism by these proteins in the context of virus infection.

In conclusion, we first show here the expression and subcellular localization of structural and accessory proteins of MERS-CoV. Furthermore, our in vitro results first identified that the M, ORF $4 \mathrm{a}$, ORF $4 \mathrm{~b}$, and ORF 5 proteins of MERS-CoV are potential interferon antagonists. ORF $4 a$ was the most potent antagonist, inhibiting multiple levels of the IFN response via the inhibition of both interferon production (IFN- $\beta$ promoter activity, IRF-3 and NF-KB activation) and the ISRE promoter element signaling pathway. To our knowledge, this is the first insight into the subcellular localization, function and pathogenic role of structural and accessory proteins of MERS-CoV. According to a recent report, the replicase polyprotein of MERS$\mathrm{CoV}$ is cleaved into 16 nonstructural proteins (van Boheemen et al., 2012); it thus remains a possibility that one or more of these products also inhibit the interferon response, much like SARS-CoV (Devaraj et al., 2007; Wathelet et al., 2007; Frieman et al., 2009; Kamitani, et al., 2009; Huang et al., 2011; Sun et al., 2012).

\section{MATERIALS AND METHODS}

Cells and virus

BHK and 293T cells were cultured in Dulbecco's modified Eagle me- 
dium (Gibco-BRL) supplemented with $10 \%$ heat-inactivated foetal bovine serum (HyClone), penicillin $(100 \mathrm{U} / \mathrm{mL})$, streptomycin $(100 \mathrm{~g} / \mathrm{mL})$, nonessential amino acids $(0.1 \mathrm{mmol} / \mathrm{L})$, and L-glutamine $(2 \mathrm{mmol} / \mathrm{L})$ (Life Technologies). Sendai virus (SeV), Cantell strain, was propagated at $37^{\circ} \mathrm{C}$ in 10 -day-old embryonated chicken eggs.

\section{Plasmid constructs}

All of the ORFs of MERS-CoV were derived from the published sequence (GenBank accession number: JX869059) and chemically synthesized (Qingke Bio-Tech Engineering Service Co., Ltd.). All genes were cloned into the pCAGGS (Niwa et al., 1991) vector constructed with an N-terminal HA tag. The signal peptide (SP) of the spike (S) protein located from 1 to 18 peptides (Lu et al., 2013). A positive control, plasmid pCAGGS-HA-NS1(PR8) encoding the NS1 protein of influenza strain PR8, was kindly provided by Dr. Georg Kochs (Department of Virology, University of Freiburg, Germany) (Kochs et al., 2007). The successful expression of the proteins was confirmed by Western blotting using a mAb against the HA tag (Anbobio). pGL3-IFNß-luc, p55CIB-luc, pNF-KB-luc, pISRE-luc, pRL-SV40, and pEGFP-IRF-3 were gifts from Zhendong Zhao (Institute of Pathogen Biology, Chinese Academy of Medical Sciences and Peking Union Medical College, China) and described elsewhere (Sun et al., 2009; Wang et al., 2013).

\section{Western blots}

293T cells were seeded in 6-well dishes and transfected with empty vector or plasmids encoding the MERS-CoV proteins. At $24 \mathrm{~h}$ posttransfection (p.t.), the cells were lysed in ice-cold RIPA buffer $(50 \mathrm{mmol} / \mathrm{L}$ Tris- $\mathrm{HCl}$ (pH 7.5), $150 \mathrm{mmol} / \mathrm{L} \mathrm{NaCl}, 1 \%$ Triton X-100, 0.1\% SDS, and $0.5 \%$ sodium deoxycholate) supplemented with a protease inhibitor mixture (Sigma). The lysates were kept on ice for $10 \mathrm{~min}$, centrifuged, and resolved by $6 \%$ SDS-PAGE. The proteins were then transferred to a PVDF membrane (Bio-Rad), blocked with $5 \%$ skim milk in PBST for $1 \mathrm{~h}$, and then probed with the indicated primary antibodies at an appropriate dilution overnight at $4^{\circ} \mathrm{C}$. The following day, the membrane was incubated with the corresponding IRDye 800-labeled IgG secondary antibodies (LI-COR Biosciences) and scanned using the Odyssey Infrared Imaging System (LI-COR Biosciences).

Indirect immunofluorescence assay and confocal microscopy

$\mathrm{BHK}$ cells were seeded onto glass coverslips in a 24-well plate and transfected with the indicated expression plasmids using the $\mathrm{X}$ tremeGENE HP reagent (Roche). At $24 \mathrm{~h}$ p.t., the cells were fixed in $4 \%$ formaldehyde, permeabilized in $0.5 \%$ Triton X-100, blocked in $5 \%$ BSA in PBS and then probed with primary antibodies for $1 \mathrm{~h}$ at room temperature. Primary antibodies used were mouse anti-HA (Anbobio), goat anti-Calnexin (Santa Cruz), goat anti-Giantin (Santa Cruz), and goat anti-ERGIC-53 (Santa Cruz). The cells were washed three times with PBS and then incubated with either donkey anti-mouse Ig conjugated with Alexa fluor 488 or donkey anti-goat Ig conjugated with Alexa fluor 594 (Molecular Probes) at a dilution of 1:500 for $1 \mathrm{~h}$. The cells were then washed and stained with 4,6-diamidino-2-phenylindole (DAPI) (Molecular Probes) to detect nuclei. To visualize GFP-IRF3 localization, 293T cells were co-transfected with pEGFP-IRF-3 and the indicated plasmids, and the MERS-CoV proteins were labeled using the primary antibody mouse anti-HA (Anbobio) and the secondary antibody TRITC-conjugated goat anti-mouse IgG. Fluorescence images were obtained and analyzed using an LSM 510 laser-scanning confocal microscope (Carl Zeiss, Oberkochen, Germany).

\section{Transfection and reporter gene assays}

293T cells were seeded in 24-well dishes and transfected with $1.5 \mu \mathrm{g}$ of the indicated plasmids, $25 \mathrm{ng}$ pRL-SV40, and $0.5 \mu \mathrm{g}$ pGL3-IFN $\beta$ luc plasmid, pNF-KB-luc plasmid or p55-CIB-luc plasmid using the $\mathrm{X}$ tremeGENE HP reagent (Roche). At $24 \mathrm{~h}$ p.t., cells were infected with Sendai virus at an $\mathrm{MOI}$ of $\sim 5$ for $18 \mathrm{~h}$. For the ISRE promoter activation assay, 293T cells were transfected with $1.5 \mu \mathrm{g}$ of the indicated plasmids, $25 \mathrm{ng}$ pRL-SV40, and $0.5 \mu \mathrm{g}$ pISRE-luc, and cells were mock treated or treated with $3,000 \mathrm{U} / \mathrm{mL}$ recombinant human IFN- $\beta$ (Calbiochem) for $18 \mathrm{~h}$. Cells were harvested, lysed, and analyzed with a DualLuciferase Reporter Assay System according to the manufacturer's protocol (Promega). Values for the samples were normalized using the Renilla luciferase values and expressed as percentages of the value for the negative control.

\section{Reverse transcription-PCR (RT-PCR)}

Total RNA was extracted from cells using TRIzol reagent (Invitrogen). RNA samples were treated with DNase I (Pierce), and reverse transcription was carried out using the Superscript cDNA synthesis kit (Invitrogen) according to the manufacturer's instructions as previously described (Lei et al., 2010). cDNA samples were subjected to PCR amplification and electrophoresis to detect IFN- $\beta$, ISG54, ISG56 expression, and the glyceraldehyde 3-phosphate dehydrogenase (GAP$\mathrm{DH}$ ) was used as an internal control to ensure that RNA of comparable quality was analyzed. The primers used were as follows: for human IFN- $\beta$, 5'-TAGCACTGGCTGGAATGAG-3' and 5'-GTTTCGGAGGTAACCTGTAAG-3'; human ISG54, 5'-CTGCAACCATGAGTGAGAA-3' and 5'-CCTTTGAGGTGCTTTAGATAG-3'; human ISG56, 5'-TACAGCAACCATGAGTACAA-3' and 5'-TCAGGTGTTTCACATAGGC-3'; and GAPDH, 5'-CGGAGTCAACGGATTTGGTCGTA-3' and 5'-AGCCTTCTCCATGGTGGTGAAGAC-3'.

\section{ACKNOWLEDGEMENTS}

We thank Dr Georg Kochs (Department of Virology, University of Freiburg, Germany) for providing pCAGGS plasmid. This work was supported by the National Basic Research Program (973 Program) (No. 2011CB504704), and the Ministry of Health of China (2014ZX10004001, 2013ZX10004601).

\section{ABBREVIATIONS}

ACE2, angiotensin-converting enzyme 2; DPP4, dipeptidyl peptidase 4; DAPI, 4,6-diamidino-2-phenylindole; ER, endoplasmic reticulum; ER$\mathrm{GIC}$, endoplasmic reticulum-Golgi intermediate compartment; GAPDH, glyceraldehyde 3-phosphate dehydrogenase; IBV, infectious bronchitis virus; IFN, interferon; IRF-3, interferon regulatory factor 3; ISG, IFN-stimulated gene; ISRE, interferon-stimulated response element; MERS-CoV, Middle East respiratory syndrome coronavirus; MHV, mouse hepatitis virus; NF-kB, neural factor $\mathrm{KB}$; ORF, open reading frame; PLP, papain-like protease; RT-PCR, Reverse transcription-PCR; SARS-CoV, severe acute respiratory syndrome-associated virus; $\mathrm{SeV}$, Sendai virus; SP, signal peptide; TGEV, transmissible gastroenteritis coronavirus 


\section{COMPLIANCE WITH ETHICS GUIDELINES}

All the authors (Yang Yang, Ling Zhang, Heyuan Geng, Yao Deng, Baoying Huang, Yin Guo, Zhengdong Zhao, and Wenjie Tan) declare that they have no conflict of interest.

This article does not contain any studies with human or animal subjects performed by the any of the authors.

\section{REFERENCES}

Basler, C.F., Wang, X., Mühlberger, E., Volchkov, V., Paragas, J., and Klenk, H.D. (2000). The Ebola virus VP35 protein functions as a type I IFN antagonist. Proc Natl Acad Sci U S A 97, 12289-12294.

Breban, R., Riou, J., and Fontanet, A. (2013). Interhuman transmissibility of Middle East respiratory syndrome coronavirus: estimation of pandemic risk. The Lancet 382, 694-649.

Corse, E., and Machamer, C.E. (2000). Infectious bronchitis virus E protein is targeted to the Golgi complex and directs release of viruslike particles. J Virol 74, 4319-4326.

Chan, R.W., Chan, M.C., Agnihothram, S., Chan, L.L., Kuok, D.I., and Fong, J.H. (2013). Tropism of and innate immune responses to the novel human betacoronavirus lineage $\mathrm{C}$ virus in human ex vivo respiratory organ cultures. J Virol 87, 6604-6614.

de Groot, R.J., Baker, S.C., Baric, R.S., Brown, C.S., Drosten, C., and Enjuanes, L. (2013). Middle East respiratory syndrome coronavirus (MERS-CoV): announcement of the Coronavirus Study Group. J Virol 87, 7790-7792.

Devaraj, S.G., Wang, N., Chen, Z., Chen, Z., Tseng, M., and Barretto, N. (2007). Regulation of IRF-3-dependent innate immunity by the papain-like protease domain of the severe acute respiratory syndrome coronavirus. J Biol Chem 282, 32208-32221.

de Wilde, A.H., Raj, V.S., Oudshoorn, D., Bestebroer, T.M., van Nieuwkoop, S., and Limpens, R.W. (2013). MERS-coronavirus replication induces severe in vitro cytopathology and is strongly inhibited by cyclosporin A or interferon- $\alpha$ treatment. J Gen Virol 94, 17491760.

Freundt, E.C., Yu, L., Park, E., Lenardo, M.J., and Xu, X.N. (2009). Molecular determinants for subcellular localization of the severe acute respiratory syndrome coronavirus open reading frame $3 \mathrm{~b}$ protein. J Virol 83, 6631-6640.

Frieman, M., Ratia, K., Johnston, R.E., Mesecar, A.D., and Baric, R.S. (2009). Severe acute respiratory syndrome coronavirus papain-like protease ubiquitin-like domain and catalytic domain regulate antagonism of IRF3 and NF-kappaB signaling. J Virol 83, 6689-6705.

Frieman, M., Yount, B., Heise, M., Kopecky-Bromberg, S.A., Palese, P., and Baric, R.S. (2007). Severe acute respiratory syndrome coronavirus ORF6 antagonizes STAT1 function by sequestering nuclear import factors on the rough endoplasmic reticulum/Golgi membrane. J Virol 81, 9812-9824.

Frieman, M., and Baric, R. (2008). Mechanisms of severe acute respiratory syndrome pathogenesis and innate immunomodulation. Microbiol Mol Biol Rev 72, 672-685.

García-Sastre, A., and Biron, C.A. (2006). Type 1 interferons and the virus-host relationship a lesson in détente. Science 312, 879-882.

Hiscox, J.A., Wurm, T., Wilson, L., Britton, P., Cavanagh, D., and Brooks, G. (2001). The coronavirus infectious bronchitis virus nucleoprotein localizes to the nucleolus. J Virol 75, 506-512.

Huang, C., Lokugamage, K.G., Rozovics, J.M., Narayanan, K., Sem- ler, B.L., and Makino, S. (2011). SARS coronavirus nsp1 protein induces template-dependent endonucleolytic cleavage of mRNAs: viral mRNAs are resistant to nsp1-induced RNA cleavage. PLoS Pathog 7, e1002433.

Kamitani, W., Huang, C., Narayanan, K., Lokugamage, K.G., and Makino, S. (2009). A two-pronged strategy to suppress host protein synthesis by SARS coronavirus Nsp1 protein. Nat Struct Mol Biol 16,1134-1140.

Khan, S., Fielding, B.C., Tan, T.H., Chou, C.F., Shen, S., and Lim, S.G. (2006). Over-expression of severe acute respiratory syndrome coronavirus $3 \mathrm{~b}$ protein induces both apoptosis and necrosis in Vero E6 Cells. Virus Res 122, 20-27.

Kochs, G., García-Sastre, A., and Martínez-Sobrido, L. (2007). Multiple anti-interferon actions of the influenza A virus NS1 protein. J Virol 81, 7011-7021.

Kopecky-Bromberg, S.A., Martínez-Sobrido, L., Frieman, M., Baric, R.A., and Palese, P. (2007). Severe acute respiratory syndrome coronavirus open reading frame (ORF) $3 \mathrm{~b}$, ORF 6 , and nucleocapsid proteins function as interferon antagonists. J Virol 81, 548-557.

Lai, M.M.C., Perlman, S., and Anderson, J.L. (2006). Coronaviridae. In:Knipe D.M., Howley P.M., eds. Fields Virology, 5th edition. Philadelphia: Lippincott Williams \& Wilkins, pp. 1305-1335.

Lei, X, Liu, X, Ma, Y, Sun, Z, Yang, Y, and Jin, Q. (2010). The 3C protein of enterovirus 71 inhibits retinoid acid-inducible gene I-mediated interferon regulatory factor 3activation and type I interferon responses. J Virol 34, 8051-8061.

Lontok, E., Corse, E., and Machamer, C.E. (2004). Intracellular targeting signals contribute to localization of coronavirus spike proteins near the virus assembly site. J Virol 78, 5913-5922.

Lu, X., Pan, J., Tao, J., and Guo, D. (2011). SARS-CoV nucleocapsid protein antagonizes IFN- $\beta$ response by targeting initial step of IFN- $\beta$ induction pathway, and its C-terminal region is critical for the antagonism. Virus Genes 42, 37-45.

Lu, G, Hu, Y, Wang, Q, Qi, J, Gao, F, and Li, Y. (2013). Molecular basis of binding between novel human coronavirus MERS-CoV and its receptor CD26. Nature 500, 227-231.

Koetzner, C.A., Kuo, L., Goebel, S.J., Dean, A.B., Parker, M.M., and Masters, P.S. (2010). MHV Accessory protein 5 a is a major antagonist of the antiviral action of interferon against murine coronavirus. $J$ Virol 84, 8262-8274.

Marra, M.A., Jones, S.J., Astell, C.R., Holt, R.A., Brooks-Wilson, A., and Butterfield, Y.S. (2003). The Genome sequence of the SARSassociated coronavirus. Science 300, 1399-1404.

McBride, R., and Fielding, B.C. (2012). The role of severe acute respiratory syndrome (SARS)-coronavirus accessory proteins in virus pathogenesis. Viruses 4, 2902-2923.

Nal, B., Chan, C., Kien, F., Siu, L., Tse, J., and Chu, K. (2005). Differential maturation and subcellular localization of severe acute respiratory syndrome coronavirus surface proteins S, M and E. J Gen Virol 86, 1423-1434.

Nieto-Torres, J.L., Dediego, M.L., Alvarez, E., Jiménez-Guardeño, J.M., Regla-Nava, J.A., and Llorente, M. (2011). Subcellular location and topology of severe acute respiratory syndrome coronavirus envelope protein. Virology 415, 69-82.

Niwa, H., Yamamura, K., and Miyazaki, J. (1991). Efficient selection for high-expression transfectants with a novel eukaryotic vector. Gene 108, 193-199. 
Raj, V.S., Mou, H., Smits, S.L., Dekkers, D.H., Müller, M.A., and Dijkman, R. (2013). Dipeptidyl peptidase 4 is a functional receptor for the emerging human coronavirus-EMC. Nature 495, 251-254.

Randall, R.E., and Goodbourn, S. (2008). Interferons and viruses: an interplay between induction, signaling, antiviral responses and virus countermeasures. J Gen Virol 89, 1-47.

Reid, S.P., Leung, L.W., Hartman, A.L., Martinez, O., Shaw, M.L., and Carbonnelle, C. (2006). Ebola virus VP24 binds karyopherin alpha1 and blocks STAT1 nuclear accumulation. J Virol 80, 5156-5167.

Shaw, M.L. 2009. Henipaviruses employ a multifaceted approach to evade the antiviral interferon response. Viruses 1, 1190-1203.

Siu, K.L., Kok, K.H., Ng, M.H., Poon, V.K., Yuen, K.Y., and Zheng, B.J. (2009). Severe acute respiratory syndrome coronavirus $M$ protein inhibits type I interferon production by impeding the formation of TRAF3.TANK.TBK1/IKKepsilon complex. J Biol Chem 284, 1620216209.

Sun, L., Xing, Y., Chen, X., Zheng, Y., Yang, Y., and Nichols, D.B. (2012). Coronavirus papain-like proteases negatively regulate antiviral innate immune response through disruption of STING-mediated signaling. PLoS One 7, e30802.

Sun, W., Li, Y., Chen, L., Chen, H., You, F., and Zhou, X. (2009). ERIS, an endoplasmic reticulum IFN stimulator, activates innate immune signaling through dimerization. Proc Natl Acad Sci U S A 106, 8653-8658.

Surjit, M., Kumar, R., Mishra, R.N., Reddy, M.K., Chow, V.T., and Lal, S.K. (2005). The severe acute respiratory syndrome coronavirus nucleocapsid protein is phosphorylated and localizes in the cytoplasm by 14-3-3-mediated translocation. J Virol 79, 11476-11486.

Susan, R.W., and Julian, L.L. (2011). Coronavirus pathogenesis. Adv Virus Res 81, 95-164.

Taylor, K.E., and Mossman, K.L. (2013). Recent advances in understanding viral evasion of type I interferon. Immunology 138, 190-197.

Totura, A.L., and Baric, R.S. (2012). SARS coronavirus pathogenesis: host innate immune responses and viral antagonism of interferon. Curr Opin Virol 2, 264-275.

van Boheemen, S., de Graaf, M., Lauber, C., Bestebroer, TM., Raj,
V.S., and Zaki, A.M. (2012). Genomic characterization of a newly discovered coronavirus associated with acute respiratory distress syndrome in humans. MBio 3, pii: e00473-12.

Wang, B., Xi, X., Lei, X., Zhang, X., Cui, S., and Wang, J. (2013). Enterovirus 71 protease 2Apro targets MAVS to inhibit anti-viral type I interferon responses. PLoS Pathog 9, e1003231.

Wathelet, M.G., Orr, M., Frieman, M.B., and Baric, R.S. (2007). Severe acute respiratory syndrome coronavirus evades antiviral signaling: role of nsp1 and rational design of an attenuated strain. J Virol 81, 11620-11633.

World Health Organization. (2013). Novel coronavirus inf1ection-update. http:// www.who.int/csr/don/2013_08_01/en/index.html.

Wurm, T., Chen, H., Hodgson, T., Britton, P., Brooks, G., and Hiscox, J.A. (2001). Localization to the nucleolus is a common feature of coronavirus nucleoproteins, and the protein may disrupt host cell division. J Virol 75, 9345-9356.

Ye, Y., Hauns, K., Langland, J.O., Jacobs, B.L., and Hogue, B.G. (2007). Mouse hepatitis coronavirus A59 nucleocapsid protein is a type I interferon Antagonist. J Virol 81, 2554-2563.

You, J., Dove, B.K., Enjuanes, L., DeDiego, M.L., Alvarez, E., and Howell, G. (2005). Subcellular localization of the severe acute respiratory syndrome coronavirus nucleocapsid protein. J Gen Virol 86, 3303-3310.

Yuan, X., Yao, Z., Shan, Y., Chen, B., Yang, Z., and Wu, J. (2005a). Nucleolar localization of non-structural protein $3 b$, a protein specifically encoded by the severe acute respiratory syndrome coronavirus. Virus Res 114, 70-79.

Yuan, X., Shan, Y., Zhao, Z., Chen, J., and Cong, Y. (2005b). G0/G1 arrest and apoptosis induced by SARS-CoV 3b protein in transfected cells. Virol J 2, 66.

Zaki, A.M., van Boheemen, S., Bestebroer, T.M., Osterhaus, A.D., and Fouchier, R.A. (2012). Isolation of a novel coronavirus from a man with pneumonia in Saudi Arabia. N Engl J Med 367, 1814-1820.

Zhao, L., Rose, K.M., Elliott, R., Van Rooijen, N., and Weiss, S.R. (2011). Cell-type-specific type I interferon antagonism influences organ tropism of murine coronavirus. J Virol 85, 10058-10068. 